\title{
A rapid technique for detection of resistance to chloramphenicol in Streptococcus pneumoniae and comparison with minimum inhibitory concentration and disk-diffusion methods
}

\author{
C. W. WALKER and D. F. J. BROWN
}

Clinical Microbiology and Public Health Laboratory, Addenbrooke's Hospital, Hills Road, Cambridge CB2 $20 W$

\begin{abstract}
Summary. Fifty-two strains of Streptococcus pneumoniae were examined for production of chloramphenicol acetyltransferase (CAT) by a rapid technique based upon induction of enzyme activity and chemical assay. This method was compared with one measuring the minimum inhibitory concentration (MIC) by agar dilution and a diffusion test with disks containing $10 \mu \mathrm{g}, 30 \mu \mathrm{g}$ and $50 \mu \mathrm{g}$ of chloramphenicol. The MIC for 13 chloramphenicol-resistant strains was $16 \mathrm{mg} / \mathrm{L}$ and for 39 sensitive strains $\leq 4 \mathrm{mg} / \mathrm{L}$. The chemical assay clearly distinguished resistant from sensitive strains; it was technically simple and provided results within $90 \mathrm{~min}$. The distinction between sensitive and resistant bacteria in the disk diffusion assay was clearer with $10-\mu \mathrm{g}$ than with $30-\mu \mathrm{g}$ and $50-\mu \mathrm{g}$ disks. However, the chemical CAT assay, with enzyme induction, is recommended when a rapid result is required.
\end{abstract}

\section{Introduction}

Chloramphenicol-resistant strains of Streptococcus pneumoniae were first described in $1970 .^{1}$ Increasing numbers of resistant strains including some with multiple resistance, have since been reported in many countries. ${ }^{2-6}$ Outbreaks of infection with chloramphenicol-resistant $S$. pneumoniae in hospitals have also occurred. ${ }^{7,8}$ Susceptibility to chloramphenicol is commonly tested by diskdiffusion methods. However, results from the UK National External Quality Assurance Scheme ${ }^{9}$ suggest that failure to detect chloramphenicol resistance in $S$. pneumoniae by disk diffusion is common. An error rate of $14.8 \%$ was reported when $25-$ or $30-\mu \mathrm{g}$ disks were used and $6.8 \%$ when $2-$ or $10-\mu \mathrm{g}$ disks were used. The clinical value of diffusion methods is also limited because the tests require overnight incubation, whereas rapid detection of resistance to chloramphenicol is desirable in lifethreatening infections such as meningitis.

Chloramphenicol resistance in $S$. pneumoniae is mediated by the production of chloramphenicol acetyltransferase (CAT), which is induced by chloramphenicol. ${ }^{10}$ Various methods for the detection of CAT in enterobacteria have been

Received 23 June 1989; accepted 8 Aug. 1989. described $^{11}$ and have been modified for rapid detection of CAT in Haemophilus influenzae. ${ }^{12,13}$ Assay of CAT in $S$. pneumoniae has been reported to be a reliable means of detecting chloramphenicol resistance, ${ }^{14}$ although the disk and tube methods used for detection of CAT did not give identical results. We have developed a technique for the rapid detection of CAT in $S$. pneumoniae based on that described for $H$. influenzae. ${ }^{13}$ It is based upon the use of microtitration trays and frozen reagents, which can be rapidly thawed for use. The main difference between the assays in $H$. influenzae and $S$. pneumoniae is the requirement for induction of CAT in $S$. pneumoniae only. ${ }^{10}$

\section{Materials and methods}

\section{Strains}

Fifty-two strains of S. pneumoniae were examined. Two chloramphenicol-resistant and 38 sensitive strains were isolated from blood and cerebrospinal fluid in the Clinical Microbiology and Public Health Laboratory, Cambridge. Eleven chloramphenicol-resistant strains and one sensitive strain were isolated in various other laboratories in the UK. All strains reported as resistant had chloramphenicol MICs of $16 \mathrm{mg} / \mathrm{L}$ when tested as described below. 


\section{MIC determination}

An agar dilution method was used. ${ }^{15}$ Twofold dilutions of chloramphenicol were prepared in Iso-Sensitest Agar (Oxoid) supplemented with defibrinated horse blood 5\%. Inocula were prepared by suspending organisms in IsoSensitest Broth and plates were inoculated by use of a multipoint inoculator (Denley). The inoculum was approximately $1 \times 10^{4} \mathrm{cfu}$. The cultures were incubated at $37^{\circ} \mathrm{C}$ for $18 \mathrm{~h}$ in an atmosphere of $\mathrm{CO}_{2} 5 \%$ in air.

\section{Disk-diffusion susceptibility tests}

Suspensions of organisms in Iso-Sensitest broth were used to flood Iso-Sensitest agar supplemented with defibrinated horse blood $5 \%$. Inocula were adjusted so that semi-confluent growth was obtained after overnight incubation of the plates. Plates were dried and disks containing $10 \mu \mathrm{g}$ (Mast), $30 \mu \mathrm{g}$ (Mast) and $50 \mu \mathrm{g}$ (Oxoid) of chloramphenicol were placed on the surface of the agar. The plates were incubated at $37^{\circ} \mathrm{C}$ for $18 \mathrm{~h}$ in an atmosphere of $\mathrm{CO}_{2} 5 \%$ in air.

\section{Induction of CAT in S. pneumoniae}

A strain of $S$. pneumoniae known to produce CAT (MQCL 1441, Division of Microbiological Reagents and Quality Control, Central Public Health Laboratory, Colindale, London) was used to determine the optimum concentration of chloramphenicol and incubation time needed to induce CAT. A bacterial suspension was made from several colonies in Todd Hewitt Broth (Difco) to a density of approximately $10^{9} \mathrm{cfu} / \mathrm{ml}$. The suspension was divided into $10-\mathrm{ml}$ portions and concentrations of chloramphenicol from 0.5 to $20 \mathrm{mg} / \mathrm{L}$ were added. The suspensions were incubated at $37^{\circ} \mathrm{C}$. At intervals, $1-\mathrm{ml}$ samples were taken and examined for CAT activity.

\section{Chemical CAT assay}

After induction, a 1-ml suspension was centrifuged in a microcentrifuge (Eppendorf) for $2 \mathrm{~min}$ and the supernate was discarded. The deposit was re-suspended in $0.2 \mathrm{ml}$ of lysing solution $(1 \mathrm{M} \mathrm{NaCl}, 0.01 \mathrm{M}$ EDTA, sodium dodecyl sulphate $0.05 \% \mathrm{w} / \mathrm{v}, \mathrm{pH} 8.0$ ) and, after incubation at $37^{\circ} \mathrm{C}$ for $15 \mathrm{~min}$, the suspension was centrifuged for $2 \mathrm{~min}$. To $50-\mu \mathrm{l}$ volumes of the supernate dispensed in duplicate wells of a microtitration tray were added $50 \mu \mathrm{l}$ of a solution containing $80 \mathrm{mM}$ Tris $\mathrm{HCl}$ buffer ( $\mathrm{pH} \mathrm{8.0),} 2 \mathrm{mM}$ acetyl coenzyme $A$ and $2 \mathrm{mM} 5,5^{\prime}$ di-thio-bis (2-nitrobenzoic acid) (DTNB); $50 \mu$ l of $5 \mathrm{mM}$ chloramphenicol were added to one well of each pair and $50 \mu \mathrm{l}$ of distilled water to the other. The tray was covered and incubated at $37^{\circ} \mathrm{C}$ in air for $5 \mathrm{~min}$. A positive reaction (i.e., CAT activity) was indicated by the development of a deep yellow colour in the well containing chloramphenicol but not in the control well with distilled water. The colour change was assessed by eye but the change in absorbance $(405 \mathrm{~nm})$ was also read by means of a microtitration plate reader (Titertek).

\section{Results}

The MICs of chloramphenicol for S. pneumoniae were bimodally distributed (fig. 1); 39 strains were sensitive to chloramphenicol (MICs $\leq 4 \mathrm{mg} / \mathrm{L}$ ) and 13 strains were resistant (MICs $16 \mathrm{mg} / \mathrm{L}$ ).

In disk-diffusion tests with $10-\mu \mathrm{g}$ disks, all the resistant strains had zone diameters of $<14 \mathrm{~mm}$, whereas with the $30-\mu \mathrm{g}$ and $50-\mu \mathrm{g}$ disks resistant strains had zone diameters of up to $18 \mathrm{~mm}$ and $19 \mathrm{~mm}$ respectively. Discrimination between sensitive and resistant populations was clearer with $10-\mu \mathrm{g}$ disks (fig. 1a) than with $30-\mu \mathrm{g}$ (fig. 1b) and $50-\mu \mathrm{g}$ disks (fig. 1c) in that the difference in zone a

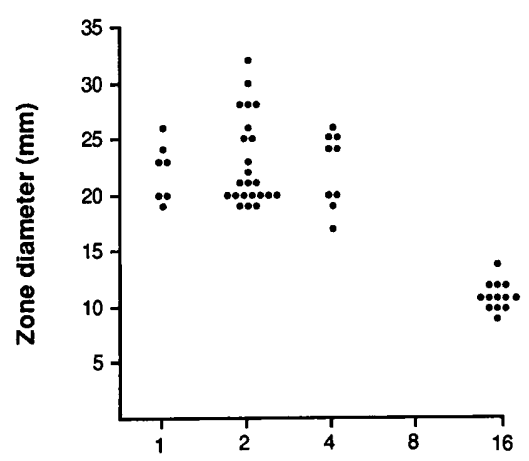

b

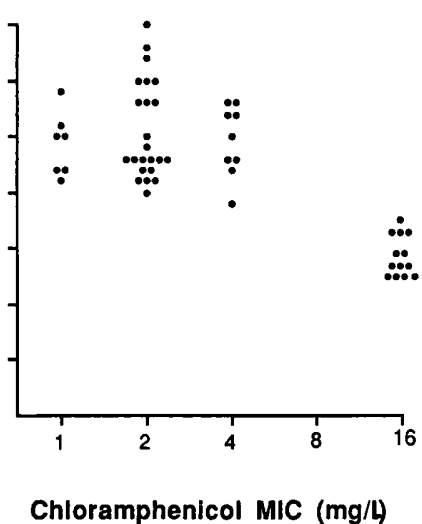

c

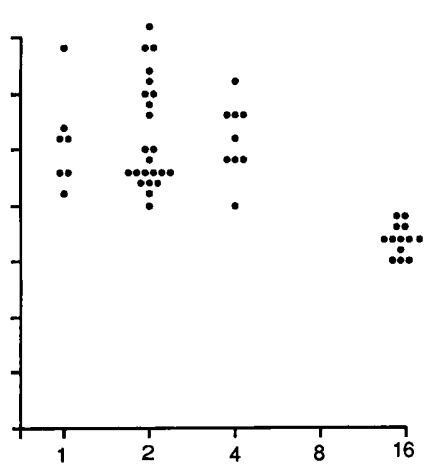

Fig. 1. The relationship of chloramphenicol MIC to zone diameter in disk-diffusion susceptibility tests with 52 strains of $S$. pneumoniae; disks contained (a) $10 \mu \mathrm{g}$, (b) $30 \mu \mathrm{g}$, and (c) $50 \mu \mathrm{g}$ of chloramphenicol. 
size between the smallest zone with a sensitive strain and the largest zone with a resistant strain was $3 \mathrm{~mm}$ with $10-\mu \mathrm{g}$ disks but only $1 \mathrm{~mm}$ with 30 $\mu \mathrm{g}$ and $50-\mu \mathrm{g}$ disks. Furthermore, the difference in mean zone diameters between sensitive and resistant populations was $11.5 \mathrm{~mm}$ with $10-\mu \mathrm{g}$ disks, $10.3 \mathrm{~mm}$ with $30-\mu \mathrm{g}$ disks and $9.1 \mathrm{~mm}$ with $50-\mu \mathrm{g}$ disks.

Induction of CAT was most rapid with chloramphenicol $2 \mathrm{mg} / \mathrm{L}$ (fig. 2). During the 2-h incubation period, the total amount of CAT produced increased with increasing concentrations up to $2 \mathrm{mg} / \mathrm{L}$, but decreased at concentrations greater than $5 \mathrm{mg} / \mathrm{L}$. Similar results were obtained when the experiment was repeated on three separate occasions. Sufficient CAT was produced after $1 \mathrm{~h}$ with chloramphenicol $2 \mathrm{mg} / \mathrm{L}$ to distinguish clearly between CAT-producing strains and non-producing strains.

In the chemical CAT assay, all the resistant strains gave a marked colour change, indicating a positive result. All the sensitive strains showed no significant colour change. Resistant strains of $S$. pneumoniae gave an increase in absorbance of $0.893-1.263$, whereas sensitive strains showed a negligible increase (0.001-0.026).

\section{Discussion}

The chloramphenicol-resistant and -sensitive bacteria could be clearly distinguished by their MIC values, as reported by others. ${ }^{2,5,6} \mathrm{We}$ found that the degree of separation of the chloramphenicol-resistant and -sensitive populations by disk diffusion depended on the chloramphenicol content of disks used. The lower the disk content, the greater was the distinction between resistant and sensitive strains. Also, with $10-\mu \mathrm{g}$ disks, all of the resistant strains had zone diameters of $\leq 14 \mathrm{~mm}$, whereas with disks of higher content, resistant strains had significantly larger zones (up to $19 \mathrm{~mm}$ in diameter with $50-\mu \mathrm{g}$ disks). These effects of disk content are similar to those observed with $H$. influenzae. ${ }^{13}$ The poorer distinction between resistant and sensitive bacteria and the larger zone sizes recorded when high content disks are used, probably explain the increased error rates with higher disk contents in the UK National External Quality Assurance Scheme. ${ }^{9}$

In the chemical assay, chloramphenicol $2 \mathrm{mg} / \mathrm{L}$ was found to be the optimum concentration for induction of CAT. Higher concentrations of chlor-

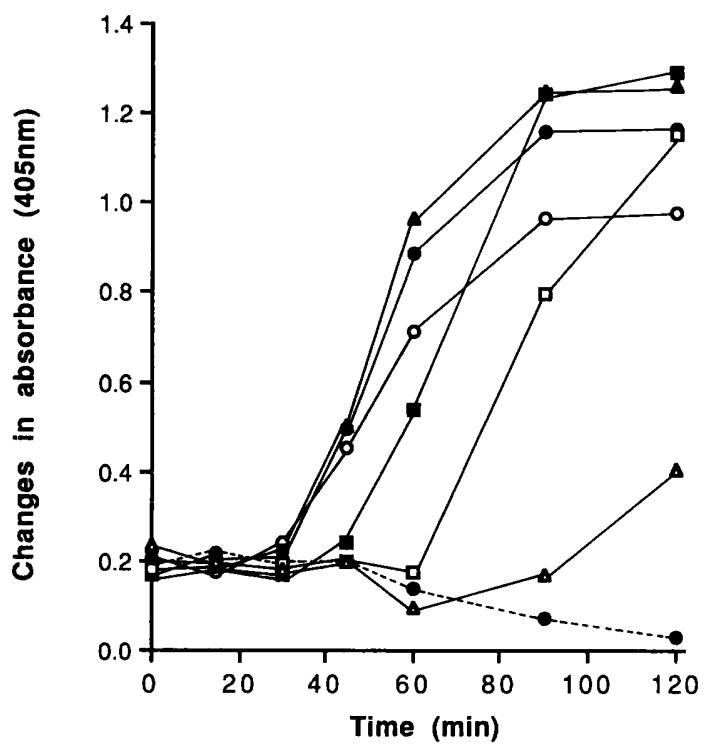

Fig. 2. The induction of CAT in $S$.pneumoniae by different concentrations $(\mathrm{mg} / \mathrm{L})$ of chloramphenicol: ---๑---, 0 ; $-\mathrm{O}-, \quad 0.5 ;-\longrightarrow, 1 ;-\boldsymbol{\Delta}, \quad 2 ; \quad-\mathbf{\square}, \quad 5$; $-\square-, 10 ;-\triangle-, 20$.

amphenicol probably inhibited protein synthesis to some extent even at sub-MIC concentrations. The longer the induction period the more CAT was produced, but after induction for $1 \mathrm{~h}$, resistant strains could be clearly distinguished from sensitive strains with the chemical CAT assay. A marked colour change indicated CAT activity in all of the resistant strains. None of the sensitive strains produced more than a very faint colour change detectable by eye. Therefore, we recommend a $1-h$ induction step before $S$. pneumoniae is tested for CAT activity. Colonies should be suspended to a density of approximately $10^{9} \mathrm{cfu} / \mathrm{ml}$ in $1 \mathrm{ml}$ of Todd Hewitt broth containing chloramphenicol $2 \mathrm{mg} / \mathrm{L}$.

Both the enzyme induction and chemical CAT assay are easy to perform and provide a result within $90 \mathrm{~min}$. The reagents for the assay can be used for both $S$.pneumoniae and $H$. influenzae except that induction is unnecessary with strains of $H$. influenzae. Reagents are stored frozen in small volumes so as to allow rapid thawing and convenient testing of single or multiple strains. In situations when a rapid chloramphenicol susceptibility test is required, colonies from primary isolation plates should be tested for CAT in this way. We are not aware of any reports of chloramphenicol-resistant, CAT-negative strains of $S$. pneumoniae. 


\section{REFERENCES}

1. Cybulska J, Jeljaszewicz J, Lund E, Munksgaard A. Prevalence of Diplococcus pneumoniae and their susceptibility to 30 antibiotics. Chemotherapy $1970 ; 15: 304-$ 316.

2. Radetsky M S, Istre G R, Johansen T L, et al. Multiply resistant pneumococcus causing meningitis: its epidemiology within a day-care centre. Lancet $1981 ; 2$ : 771773.

3. Appelbaum P C, Bhamjee A, Scragg J N, Hallett A F, Bowen A J, Cooper R C. Streptococcus pneumoniae resistant to penicillin and chloramphenicol. Lancet 1977; 2: 995-997.

4. Latorre C, Juncosa T, Sanfeliu I. Antibiotic resistance and serotypes of 100 Streptococcus pneumoniae strains isolated in a children's hospital in Barcelona, Spain. Antimocrob Agents Chemother 1985; 28 : 357-359.

5. Appelbaum P C. World-wide development of antibiotic resistance in pneumoccocci. Eur J Clin Microbiol 1987; 6: $367-377$.

6. George R C, Cooper P G, Erdman Y J. Not the first multiresistant pneumococcus in Britain. $\mathrm{Br}$ Med $\mathrm{J}$ 1987; 295: 1208.

7. Gould F K, Magee J G, Ingham H R. A hospital outbreak of antibiotic-resistant Streptococcus pneumoniae. J Infect 1987 ; 15 : 77-79.

8. Moore E P, Williams E W. Hospital transmission of multiply antibiotic-resistant Streptococcus pneumoniae. J Infect 1988; 16: 199-200.

9. Snell J J S, George R C, Perry S F, Erdman Y J. Antimicrobial susceptibility testing of Streptococcus pneumoniae: quality assessment results. $J$ Clin Pathol $1988 ; 41$ : 384-387.

10. Dang-Van A, Tiraby G, Acar J F, Shaw W V, Bouanchaud D H. Chloramphenicol resistance in Streptococcus pneumoniae: enzymatic acetylation and possible plasmid linkage. Antimicrob Agents Chemother 1978; 13: $577-583$.

11. Shaw W V. Chloramphenicol acetyltransferase from chloramphenicol-resistant bacteria. Methods Enzymol 1975; 43: 737-755.

12. Azemun P, Stull T, Roberts M, Smith A L. Rapid detection of chloramphenicol resistance in Haemophilus influenzae. Antimicrob Agents Chemother $1981 ; 20$ : 168-170.

13. Walker C W, Brown D F J. The reliability of methods for detecting chloramphenicol resistance in Haemophilus influenzae. J Antimicrob Chemother 1988; 22 : 905-910.

14. Matthews H W, Baker C N, Thornsberry C. Relationship between in vitro susceptibility test results for chloramphenicol and production of chloramphenicol acetyltransferase by Haemophilus influenzae, Steptococcus pneumoniae and Aerococcus species. J Clin Microbiol $1988 ; 26$ : 2387-2390.

15. Ericsson $\mathbf{H} \mathbf{M}$, Sherris J C. Antibiotic sensitivity testing. Report of an International Collaborative Study. Acta Pathol Microbiol Scand. 1971; Section B: Suppl 217. 